[white paper]

Diamond Open Access

\title{
Três conectivos matemáticos
}

\author{
Colaboração Matemática Aberta1 \\ 25 de Janeiro de 2021
}

\begin{abstract}
Resumo
Apresentamos os três conectivos mais básicos da matemática formal (e, ou, não) e suas implicações nas respectivas proposições.
\end{abstract}

palavras-chave: matemática básica, lógica, conectivos

A versão mais atualizada deste artigo está disponível em https://osf.io/4ukf3/download

\section{Introdução}

1. O objetivo deste artigo é familiarizar o leitor não especialista nos mais básicos e fundamentais conectivos da matemática.

2. Esses livros são excelentes referências para iniciar os estudos neste maravilhoso universo das demonstrações matemáticas [1-3].

3. Este artigo faz parte da série Demonstrações Matemáticas [4,5].

4. Para estudar/revisar conceitos de Matemática Básica, veja [6-9].

${ }^{1}$ Todos os autores com suas afiliações aparecem no final deste artigo. 


\section{Conectivos}

5. Na tabela a seguir estão os três conectivos matemáticos.

6.

$\begin{array}{cc}\text { símbolo } & \text { significado } \\ \vee & \text { OU } \\ \wedge & \text { E } \\ \neg & \mathrm{NA} O\end{array}$

\section{Proposições}

7. Sejam $P$ e $Q$ proposições.

8. Uma proposição pode ser uma sentença do tipo José foi à padaria ou uma fórmula matemática.

\section{Conectando as proposições}

9. A proposição $P \vee Q$ significa " $P$ ou $Q$ " e é denominada disjunção de $P$ e $Q$.

10. A proposição $P \wedge Q$ significa " $P \underline{\text { e }} Q$ " e é denominada conjunção de $P$ e $Q$.

11. $\neg P$ é a negação de $P$.

12. Se $P$ for verdadeiro, $\neg P$ é falso.

13. Se $P$ for falso, $\neg P$ é verdadeiro. 


\section{Notação}

14. Por convenção, temos
(a) $\neg P \wedge Q=(\neg P) \wedge Q$,
(b) $\neg P \wedge Q \neq \neg(P \wedge Q)$.

\section{Considerações Finais}

15. Note que, enquanto os conectivos $\vee$ e $\wedge$ conectam duas proposições, a negação $(\neg)$ está ligada a apenas uma proposição.

\section{Ciência Aberta}

O arquivo latex para este artigo, juntamente com outros arquivos suplementares, estão disponíveis em [10]. Seja coautor(a) deste artigo, envie sua contribuição para mplobo@uft.edu.br.

\section{Referências}

[1] Velleman, Daniel J. How to prove it: A structured approach. Cambridge University Press, 2019.

[2] Warner, Steve. Pure Mathematics for Beginners. GET 800, 2018.

[3] Warner, Steve. Abstract Algebra for Beginners. GET 800, 2018.

[4] Lobo, Matheus P. "N E 2 Elevado a N Menos 1 Não Primos." OSF Preprints, 23 Jan. 2021.

https://doi.org/10.31219/osf .io/k67em

[5] Lobo, Matheus P. "Existem Infinitos Primos." OSF Preprints, 24 Jan. 2021. https://doi.org/10.31219/osf .io/nz7q3 
[6] OJMP Brasil. "Fundamentos da Matemática". https://ojmpbr. wordpress. com/fundamentos-da-matematica

[7] Lobo, Matheus P. "Matemática Minimalista: Menos É Mais." OSF Preprints, 18 Oct. 2020.

https://doi.org/10.31219/osf .io/pey6z

[8] Lobo, Matheus P. "Matemática Zero." OSF Preprints, 1 Oct. 2020. https://doi.org/10.31219/osf .io/dgsf2

[9] Lobo, Matheus P. "Para Que Servem Os Números?." OSF Preprints, 8 Oct. 2020. https://doi.org/10.31219/osf.io/tyn7k

[10] Lobo, Matheus P. "Open Journal of Mathematics and Physics (OJMP)." OSF, 21 Apr. 2020.

https://doi.org/10.17605/osf .io/6hzyp

\section{Colaboração Matemática Aberta}

Matheus Pereira Lobo (autor principal, mplobo@uft.edu.br) $)^{1,2}$ https://orcid.org/0000-0003-4554-1372

${ }^{1}$ Universidade Federal do Tocantins (Brasil)

${ }^{2}$ Universidade Aberta (UAb, Portugal) 